\title{
Protocol and Rationale-The Efficacy of Minocycline as an Adjunctive Treatment for Major Depressive Disorder: A Double Blind, Randomised, Placebo Controlled Trial
}

\author{
Olivia May Dean ${ }^{1,2,3}$, Michael Maes ${ }^{4}$, Melanie Ashton ${ }^{1}$, Lesley Berk ${ }^{4}$, Buranee Kanchanatawan ${ }^{5}$, Atapol \\ Sughondhabirom ${ }^{5}$, Sookjareon Tangwongchai ${ }^{5}$, Chee Ng ${ }^{6}$, Nathan Dowling ${ }^{6}$, Gin S. Malhi ${ }^{7,8}$, Mlchael Berk ${ }^{1,2,3,9,10}$ \\ 'Innovations in Mental and Physical Health and Clinical Treatments Strategic Research Centre, Deakin University, School of Medicine, \\ Geelong, ${ }^{2}$ Florey Institute for Neuroscience and Mental Health, University of Melbourne, Parkville, ${ }^{3}$ Department of Psychiatry, University \\ of Melbourne, Parkville, Australia, ${ }^{4}$ Department of Psychiatry, Chulalongkorn University, Bangkok, Thailand, ${ }^{5}$ Centre for Mental Health \\ and Wellbeing Research, Deakin University, School of Psychology, Burwood, ${ }^{6}$ Department of Psychiatry, University of Melbourne, The \\ Melbourne Clinic, Richmond, ${ }^{7}$ Discipline of Psychiatry, Sydney Medical School, University of Sydney, Sydney, ${ }^{8}$ Department of Psychiatry, \\ CADE Clinic, St. Leonards, ${ }^{9}$ Orygen Youth Health Research Centre, Parkville, ${ }^{10}$ Centre of Youth Mental Health, University of Melbourne, \\ Parkville, Australia
}

\begin{abstract}
While current pharmacotherapies are efficacious, there remain a clear shortfall between symptom remission and functional recovery. With the explosion in our understanding of the biology of these disorders, the time is ripe for the investigation of novel therapies. Recently depression is conceptualized as an immune-inflammatory and nitro-oxidative stress related disorder. Minocycline is a tetracycline antibiotic that has anti-inflammatory, pro-oxidant, glutamatergic, neurotrophic and neuroprotective properties that make it a viable target to explore as a new therapy. This double blind, randomised, placebo controlled adjunctive trial will investigate the benefits of $200 \mathrm{mg} /$ day of minocycline treatment, in addition to any usual treatment, as an adjunctive treatment for moderate-severe major depressive disorder. Sixty adults are being randomised to 12 weeks of treatment (with a 4 week follow-up post-discontinuation). The primary outcome measure for the study is mean change on the MontgomeryAsberg Depression Rating Scale (MADRS), with secondary outcomes including the Social and Occupational Functioning Assessment Scale (SOFAS), Clinical Global Impressions (CGI), Hamilton Rating Scale for Anxiety (HAM-A), Patient Global Impression (PGI), Quality of Life Enjoyment and Satisfaction Questionnaire (Q-LES-Q) and Range of Impaired Functioning Tool (LIFE-RIFT). Biomarker analyses will also be conducted at baseline and week 12. The study has the potential to provide new treatment targets, both by showing efficacy with a new class of 'antidepressant' but also through the analysis of biomarkers that may further inform our understanding of the pathophysiology of unipolar depression.
\end{abstract}

KEY WORDS: Minocycline; Depression; Clinical research protocol; Inflammation; Oxidative stress.

\section{INTRODUCTION}

The World Health Organisation's Global Burden of Disease report cited major depression as the fourth leading cause of disease burden, as measured by disability-adjusted life years, and the leading cause in terms of years lived with disability. ${ }^{1)}$ This infers that major depression is

\footnotetext{
Received: March 21, 2014 / Revised: April 29, 2014

Accepted: April 30, 2014

Address for correspondence: Olivia May Dean, PhD Innovations in Mental and Physical Health and Clinical Treatments Strategic Research Centre, Deakin University, School of Medicine, Kitchener House, P.O. Box 281, Geelong VIC 3220, Australia

Tel: +61-3-4215-3300, Fax: +61-3-4215-3491

E-mail: oliviad@barwonhealth.org.au

Trial registration: This trial is registered on the Australian and New Zealand Clinical Trial Registry: ACTRN12612000283875.
}

possibly one of the most protracted and disabling illness, associated with great societal costs, of all modern medical conditions. A pertinent factor intertwined with such a crippling course is the overall poor treatment outcome for major depressive disorder (MDD). Despite having been traditionally viewed with a comparatively favourable prognosis within psychiatric disorders, remission and functional recovery are uncommon in practice.

One of the many factors underlying the poor treatment outcome of MDD is that, by and large, it is a syndrome of heterogeneous and imperfectly understood aetiology. Traditionally, MDD was believed to be caused by a dysfunction of neurotransmission, focused initially on the serotonergic, and later the noradrenergic pathways however recently it has been postulated that while alterations in

(c) This is an Open-Access article distributed under the terms of the Creative Commons Attribution Non-Commercial License (http://creativecommons.org/licenses/by-nc/3.0) which permits unrestricted non-commercial use, distribution, and reproduction in any medium, provided the original work is properly cited 
neurotransmitter levels and function are present (including glutamate and gamma-aminobutyric acid [GABA] in addition to serotonin and noradrenaline), these may not be the primary aetiological basis of the disorder. Indeed, this notion is supported by the lack of complete efficacy for up to $30 \%$ of people with MDD by treatment with serotonin and noradrenaline reuptake inhibitors, directly effecting neurotransmitter levels in the brain. ${ }^{2)}$

Activation of immune-inflammatory pathways including changes in pro-inflammatory and cell-mediated immune (CMI) cytokines have been documented extensively in MDD. Increased levels of pro-inflammatory and CMI cytokines including interleukin-1 (IL-1), IL-2, IL-6, IL-8, IL-12, interferon- $\gamma$ (IFN- $\gamma$ ) and tumour necrosis factor- $\alpha$ (TNF- $\alpha)^{2)}$ are reported in MDD. Conversely, treatment with cytokines such as IFN- $\alpha$ have been related to decreased mood and increased depressive symptoms. ${ }^{3)}$ Antidepressant treatments are believed to reduce the imbalanced cytokine levels in MDD. While there is, at present, a paucity of studies directly investigating changes pre- and post-antidepressant treatment, there is evidence to suggest that some antidepressants normalize cytokine levels and this may be an additional therapeutic target, secondary to their effects on neurotransmitters. ${ }^{4)}$

In depression, the role of nitro-oxidative stress has been explored in a small collection of studies. Peet et al. ${ }^{5)}$ presented evidence of depleted levels of omega-3-fatty acids in MDD, while Maes et al. ${ }^{6,7)}$ showed a significantly lowered oxidative potential index in fatty acids and lower concentrations of serum vitamin $\mathrm{E}$, a fat-soluble antioxidant, in MDD compared with healthy volunteers. Bilici et al. ${ }^{8)}$ showed decreased levels of the antioxidant enzyme, glutathione peroxidase. Similarly, the lack of adequate antioxidant defence in MDD is evidenced by increases in oxidative stress markers. Lipid peroxidation products have been found to be significantly higher in MDD when compared to controls. ${ }^{8,9)}$ Furthermore, these alterations may return to normal after antidepressant treatment. ${ }^{8,10)}$ Higher serum levels of 8-hydroxy-2'-deoxyguanosine (8-OhdG), a biomarker of DNA damage, were found in those with MDD than in controls. ${ }^{11)}$ The levels of inducible nitric-oxide (NO) synthase (iNOS) are higher in depression, while there is evidence of hyper-nitrosylation, the formation of NO-adducts. ${ }^{12,13)}$ Individually, these studies are limited by their small sample sizes and restricted focus of attention, but they conglomerate to provide cogent support for the oxidative stress hypothesis for depression.

Finally, one of the other major targets emerging in discovering the underlying aetiology of MDD are alterations generally known as neuroprogression; that is lowered neurogenesis and neuroplasticity, cell loss and activation of apoptotic. ${ }^{14)}$ There are several identified neurotrophins in the brain that have been investigated in MDD. These include, brain-derived neurotrophic factor (BDNF), neurotrophin-3 (NT-3), and glial cell line-derived neurotrophic factor. In depression, these factors have been shown to be reduced and this may contribute the pathophysiology of the disorder. ${ }^{15,16)}$ Furthermore, these neurotrophins are influenced by oxidative stress and inflammatory mediators, thereby creating an integrated system of pathophysiology that may underlie depression. Similar to inflammatory changes, alterations in neurotrophins have been found to be somewhat reversed following treatment and remission of symptoms. ${ }^{15,16)}$ Together, these changes in oxidative inflammatory and neurotrophic factors are thought to play a pivotal role in the process of neuroprogression that is operative in mood disorders. ${ }^{17}$

Minocycline has been shown to have properties that may address these pathologies in depression. Minocycline has been shown to have antioxidant properties, making it appealing as a potential treatment for depression given the perturbations in antioxidant defences in MDD. ${ }^{18)}$ In terms of reducing free radicals, minocycline has been shown to dose-dependently decrease nitrite levels and nitric oxide synthase expression following lipopolysaccaride (LPS) treatment. ${ }^{19)}$ Rise in NO levels in response to 3-nitropropioic acid (3-NP; an inhibitor of the electron transport enzyme succinate dehydrogenase) was also attenuated using minocycline. ${ }^{20)}$

Similarly, minocycline was shown to attenuate increases in malondialdehyde (a marker of lipid peroxidation), following 3-NP treatment. ${ }^{20)}$ Lipid peroxidation and free radical generation were also shown to be decreased in ischemic mouse forebrain following minocycline treatment. ${ }^{21)}$ Furthermore, minocycline has been shown to prevent increases in antioxidants in response to oxidative stress. In rat brain homogenates, minocycline was found to reduce increases in catalase and succinate dehydrogenase following 3-NP treatment. ${ }^{20)}$ Conversely, minocycline treatment has not been shown to affect the reduced glutathione/oxidised glutathione (GSH/GSSG) ratio following traumatic brain injury in adult mice. ${ }^{22)}$

Minocycline has also has reported anti-inflammatory effects that are not related to its microbial properties, but rather due to its purported inhibition of microglial activation. High dose minocycline is reported to reduce IL-1 $\beta$ levels following traumatic brain injury. ${ }^{22)}$ This has also been replicated in MDMA-treated rats. ${ }^{23)}$ Minocy- 
cline has also been found to reduce prostaglandin- $E_{2}$ $\left(\mathrm{PGE}_{2}\right)$ and inhibit COX-2 expression in a dose-dependent manner. ${ }^{19)}$ Rises in TNF- $\alpha$ levels were also attenuated following minocycline treatment following administration of 3-NP. ${ }^{20)}$ These factors are especially relevant to MDD where increased levels of cytokines are reported.

In MDD there are reported decreases in neurotrophic factors such as BDNF and Bcl-2. Minocycline has been shown to significantly increase Bcl-2 RNA expression both alone, and in the presence of oxidative stress (hydrogen peroxide treatment). ${ }^{24)}$ Similarly, minocycline has been shown to increase nerve growth factor-induced PC12 neuronal growth in a dose-dependent manner. ${ }^{25)}$

Minocycline has also been shown to be neuroprotective by preventing cell loss. In mouse models of traumatic brain injury, minocycline has been shown to reduce lesion volume. $^{21,26)}$ In one study modelling cerebral ischemia, minocycline was shown to reduce inflammation, but also decreased neurogenesis (shown by BrdU staining). ${ }^{27}$ ) However, this has been disputed in a similar study showing minocycline increased BrdU expression and reduced microglia activation. ${ }^{28)}$ Dosing and occlusion strategies may explain differences in results. Using hydrogen peroxide to attenuate insulin-like growth factor-1-promoted neuronal survival, minocycline was shown to prevent this loss of neuronal survival. ${ }^{29)}$ Minocycline is also reported to prevent excitotoxic-induced cell death following NMDA treatment. ${ }^{30)}$ The latter is especially relevant to the current study, as changes in glutamate pathways have been reported in MDD.

Following glutamate treatment, minocycline-treated rat cortical neurons were found to have increased cell viability compared to controls, indicating decreased glutamate-induced neurotoxicity. ${ }^{21,31)}$ Studies have found reductions in MK-801-induced disruptions in prepulse inhibition, and both N-methyl-D-aspartate (NMDA) antagonist- and methamphetamine-induced hyperlocomotion (two behavioural models associated with psychotic symptoms) in mice treated with minocycline. ${ }^{32,33)}$

There is preclinical data indicating benefits of minocycline in models investigating depressive-like behaviour. In a study involving both independent and co-administration of minocycline in rats, minocycline was shown to be effective in the Porsolt swim test. Sub-threshold doses were of both current antidepressants and glutamate antagonists in combination with sub-threshold doses of minocycline were also shown to have antidepressant effects. ${ }^{34)}$ Conversely, in a study involving adult male SpragueDawley rats, the same dose of minocycline was shown to have no effect on swim times in the Porsolt swim test. ${ }^{35)}$

In a more recent study, learned helplessness was used as a model of depression and intracerebroventricular minocycline treatment was explored. The study found that rats treated with minocycline had improved performance on the conditioned avoidance test (mild foot shock) and this did not correlate to changes in locomotor activity. This suggests the effects of minocycline are anti-depressant. The study found biological correlates with serotonin turnover in the orbitofrontal cortex; being increased in rats with learned helplessness and this was not altered by minocycline treatment. However, there were no changes to serotonin in other brain regions investigated. No changes to dopamine levels were reported although the data suggested decreased dopamine levels in rats who acquired learned helplessness. Interestingly, although no deficit in dopamine was reported, minocycline caused an increase in dopamine levels in the amygdala. Finally, BDNF levels were reduced following the acquisition of learned helplessness and these remained unchanged following minocycline treatment. $^{36)}$

Taken together these data regarding the mechanisms of action of minocycline mirror many of the deficits seen in MDD. It is not surprising that minocycline has already been put forward as a potential antidepressant, ${ }^{37)}$ but what is surprising is that to date, no clinical trials have been performed, or are currently registered with the major clinical trials registry.

We have reviewed the literature regarding minocycline's use, to date, in psychiatry. ${ }^{18)}$ Initial interest began with case reports in schizophrenia, reporting serendipitous reductions in psychotic symptoms following minocycline treatment. ${ }^{38)}$ Following on from these findings, the same research group conducted an open label study of 22 people diagnosed with schizophrenia. These participants were given $300 \mathrm{mg} /$ day of minocycline and were shown to have reduced symptoms after eight weeks of treatment. These participants had either been treatment resistant in the past or were currently not responding to treatment. No adverse events were reported following the eight-week treatment phase. ${ }^{39)}$ The first double blind, placebo controlled trial in schizophrenia investigated recently diagnosed ( $<5$ years) participants (minocycline: $n=36$, control: $n=18)$ who were currently experiencing symptoms (Positive and Negative Syndrome Scale [PANSS] > 60). Participants were assigned to either $200 \mathrm{mg}$ /day of minocycline or placebo in addition to an atypical antipsychotic medication for a total of 22 weeks. Results showed improvements in negative and cognitive symptoms, as well 
as overall improvements in functioning. ${ }^{40)}$

Specific to depression, a case report regarding an individual with bipolar disorder who has predominantly depressive episodes was reported by Levine et al. ${ }^{41)}$ While admitted as an inpatient, the individual received 150 $\mathrm{mg}$ /day of minocycline and within four days had reported an attenuation of depressive symptoms. These improvements remained following withdrawal of minocycline treatment. ${ }^{41)}$ To provide further clinical support for the proposed trial, an open-label study has been conducted in a cohort of participants diagnosed with psychotic depression (major depression with psychotic features). After 6 weeks of treatment with minocycline $(150 \mathrm{mg} /$ day $) \mathrm{im}-$ provements were not only seen in psychotic symptoms, but dramatic reductions in depression symptoms were also reported. Based on both the biochemical indication and the clinical observations, minocycline may have antidepressant effects. The current study aims to explore the potential of minocycline as an adjunctive treatment for unipolar depression.

\section{Aims}

Using a randomised placebo controlled trial, we aim in this pilot study to test the efficacy of adjunctive minocycline (100 mg twice daily) in the acute treatment of unipolar depression to determine if:

1. Twelve weeks treatment of $200 \mathrm{mg} /$ day minocycline treatment reduces severity of depression compared to individuals taking treatment as usual: Primary aim.

2. Twelve weeks with $200 \mathrm{mg}$ /day minocycline treatment will improve self-reported symptom burden, quality of life, overall functioning and clinical impression and reduce symptoms of anxiety.

3. There are continued benefits following cessation of trial treatment assessed at 4 weeks following discontinuation.

4. Whether 12-weeks treatment with $200 \mathrm{mg}$ /day minocycline reduces serum markers of inflammation.

5. Whether the change in immune-inflammatory and nitro-oxidative markers correlates with change in depressive symptomatology.

\section{Primary Hypothesis}

1. Twelve-weeks of adjunctive minocycline treatment will be superior to placebo for reducing symptoms of depression using the Montgomery-Asberg Depression Rating Scale (MADRS).

\section{Secondary Objectives/Hypotheses}

2. Twelve weeks of adjunctive minocycline treatment will be superior to placebo for reducing clinical global status, and improving quality of life and functioning based on validated rating scales.

3. Those who were taking the minocycline treatment will have better outcomes 4 weeks post-treatment discontinuation, based on symptomology, quality of life and functioning than those taking the placebo.

4. Those taking minocycline treatment will have reduced levels of inflammatory and oxidative stress (lipid peroxidation) levels in peripheral (blood) samples.

5. Clinical change will correlate with discernible changes in inflammatory and oxidative stress levels in the peripheral sample.

\section{METHODS}

\section{Study Design}

This is a 12-week, parallel group, double-blind, randomised-control trial in which 60 participants with moderate-to-severe MDD will be allocated receive either 200 $\mathrm{mg}$ /day minocycline or placebo, in addition to treatment as usual (medication, psychotherapy or any other treatment).

Assessments are completed at baseline and weeks 0, 2, 4, 8 and 12. A follow-up (post-treatment discontinuation) assessment is completed at week 16. Randomisation is carried out according to the International Conference on Harmonisation of Technical Requirements for Registration of Pharmaceuticals for Human Use (ICH) guidelines by an independent researcher. Participants are allocated to treatment groups using randomly permuted blocks $(4 \times 2)$ to maintain approximately equal group sizes over time.

\section{Identity of Investigational Product and Comparators}

Minocycline: $200 \mathrm{mg} /$ day of minocycline (given as 100 mg twice daily) will be used in the study.

Placebo: Placebo tablets (starch) matched in appearance and taste will be used.

\section{Setting}

The current study is being led by Dr. Olivia Dean from Deakin University, in collaboration with Professor (Prof.) Michael Berk from Deakin University/Barwon Health, Prof. Chee $\mathrm{Ng}$ from the Univesrity of Melbourne/The Melbourne Clinic and Profs. Michael Maes and Buranee 
Kanchanatawan from Chulalongkorn University. The study will be conducted at three sites; Barwon Health and The Melbourne Clinic, both based in Victoria, Australia and Chulalongkorn University, Bangkok, Thailand.

\section{PROCEDURE}

\section{Selection of Study Population}

Potential participants are identified through referral by both the private and public treating sectors and by community advertisements. Participants expressing interest in the trial are contacted and invited to participate in a screening interview. Informed consent is obtained at the initial interview, prior to any study procedures commencing.

\section{Inclusion criteria}

For inclusion in the study, participants must fulfil all of the following criteria:

- Aged 18 years or over

- Capacity to consent to the study and to follow its instructions and procedures

- Fulfil the Diagnostic and Statistical Manual of Mental Disorders 4th edition, text revision (DSMIV-TR) diagnostic criteria for MDD, single episode or recurrent scoring 25 or over on the MADRS, at the baseline visit

- Participants on antidepressant therapy need to have been on the same treatment for at least two weeks prior to randomisation

- Utilising effective contraception (other than the contraceptive pill due to drug interactions) if female of child-bearing age and sexually active

\section{Exclusion criteria}

Participants are ineligible to enter the trial under the following conditions:

- Concurrent diagnosis of bipolar disorder I, II or not otherwise specified

- Three or more failed adequate trials of antidepressant therapy for the current major depressive episode or electroconvulsive therapy for the current major depressive episode

- Known or suspected clinically unstable systemic medical disorder (including a pre-existing infectious illness requiring tetracycline antibiotic treatment)

- Pregnancy or breastfeeding

- Contraindications to tetracyclines, including allergy or other intolerance; prior tetracycline use within 2 months of baseline visit

- Current treatment with $>5 \mathrm{mg}$ beta caretone or $>300 \mu \mathrm{g}$ retinol equivalent

- Current treatment with isotretinoin or etretinate

- Current treatment with anticoagulants (excluding aspirin)

- Current treatment with methoxyflurane

- Current treatment with penicillin

- Females of childbearing age currently relying solely on the contraceptive pill as contraception

- Current enrolment in another clinical trial

- Participants requiring treatment with antacids containing aluminium, calcium or magnesium and preparations containing iron must take these preparations at least 2 hours before or after minocycline administration. This should be clearly stated at each visit.

Withdrawal of patients from treatment or assessment

Participants will be withdrawn from the trial under the following conditions:

- Failure to take the trial medication for seven consecutive days

- Cessation of effective contraception or confirmed pregnancy

- Withdrawal of consent

- Emergence of serious adverse events suspected to be associated with the trial medication

- Unavoidable commencement on anticoagulants (other than aspirin), $>5 \mathrm{mg}$ beta caretone or $>300 \mu \mathrm{g}$ retinol equivalent, isotretinoin or etretinate, the contraceptive pill, methoxyflurane or penicillin.

- Participants who require tetracycline antibiotic treatment during the course of the study will be withdrawn.

Note: In cases of the emergence of adverse effects of minocycline treatment (such as vertigo), trial medication will be stopped for five days and then recommenced. Participants will be contacted two days following recommencement to determine if adverse effects have returned.

\section{Randomisation - Method of Assigning Patients to Treatment Groups}

After the baseline assessment has been completed, the participant will be randomised to one of the two treatment groups. Randomisation is enabled by a computer-based random number generator. Participating pharmacies have the randomisation code, to unblind participants if required. The randomisation will be double-blind and only 
the pharmacist will know which group the participant has been allocated to. Blindness will be maintained by ensuring that the packaging, appearance and colour of minocycline and placebo capsules are identical. Additionally, a third blinding aspect has been included, whereby all data analysis conducted by the statistician is conducted blind to treatment arms.

Participants are randomised strictly sequentially, as they are eligible for randomisation. Randomisation schemes are organised and administered centrally by the Innovations in Mental and Physical Health Aand Clinical Treatments Strategic Research Centre (IMPACT SRC). Each participant will have a unique identification number (Participant ID\#) for anonymous data analysis.

\section{Accountability}

The study treatments will only be used as directed in the protocol. From visit 1, participants will be requested to return all unused investigational products and empty containers to the investigator or research assistants. Records of overall dispensing and returns will be calculated and documented by the pharmacist involved in the study. Adherence to medication will be assessed by self-report and a pill count. This data will be used to calculate compliance with medication for analysis purposes.

\section{VARIABLES AND INSTRUMENTS}

\section{Assessment of Inclusion and Exclusion Criteria}

- Unipolar depression (and the absence of bipolar disorder) will be confirmed using the Mini International Neuropsychiatric Interview for DSM-IV (MINIPlus) version 5.

- Depressed severity will be established with the MADRS (moderate-to-severe depression defined as MADRS $\geq 25$ ).

- Stability of current treatment and use of previous antidepressants and contraindicated medications will be assessed at the screening visit by the researcher based on questions in the case report form.

- Urine pregnancy tests will be conducted on females of child-bearing age to exclude pregnancy.

\section{Enrolment and Demographic Variable(s)}

The following variables will be assessed for enrolment in the study and for demographic purposes:

- Medical history

- Previous medication

- Basic physical examination (pregnancy test for fe- males, height and weight)

- Family history

- Demographics (age, gender, socioeconomic status, educational attainment)

\section{Efficacy Measure(s)}

A battery of validated outcome measures, focussing on both depressive symptomatology and global clinical and functional status, will be used at baseline and at weeks 2 , 4, 8 and 12 post-baseline intervals. Efficacy measures include:

- MADRS (primary outcome)

- Clinical Global Impression - Improvement (CGI-I) and - Severity (CGI-S) scales

- Patient Global Impression - Improvement (PGI-I) scale

- Hamilton Anxiety Rating Scale (HAMA)

\section{Functioning and Quality of Life Measures}

- Quality of life will be assessed using the Quality of life Enjoyment and Satisfaction Questionnaire Short Form (Q-LES-Q-SF). We will look at change in score over time at 12 and 16 weeks.

- The Social and Occupational Functioning Scale (SOFAS) will be used to measure of psychosocial (social and occupational) functioning over time at 12 and 16 weeks.

- The Range of Impaired Functioning Tool (LIFE-RIFT) will also be used to measure levels of functioning over time at 12 and 16 weeks.

\section{Predictors and Moderators of Response}

- The Standardised Assessment of Personality-Abbreviated Scale (SAPAS) will be used to identify those with suspected personality disorders and this will be used as a covariate in the analysis and also used as a variable of interest in examining treatment response.

- Analysis of biomarkers of oxidative and inflammatory markers will be used to determine how treatment response (if present) correlates to changes in biological markers.

- Changes in biomarkers will be used to explore the underlying pathophysiology of depression.

\section{Assessment of Safety}

Side-effects will be assessed with open questions and recorded using the standardised adverse events sheet included in the CRF. 


\section{Biomarkers}

Participants will also be asked to provide two blood samples for research analysis. The provision of the blood samples is optional and does not affect participation in the clinical trial. These samples will be collected at baseline and the end of the treatment phase (week 12) and will involve the collection of a 3 tubes of blood (approximately $30 \mathrm{ml}, 2$ tablespoons) at each collection. This sample will be processed (centrifuged and aliquoted into $500 \mu 1$ aliquots) and frozen at $-80^{\circ} \mathrm{C}$ until the time of analysis

The research sample will be collected to investigate inflammatory and oxidative stress markers to determine if and how these treatments are impacting at a biological level (in addition to the subjective perception of symptom change). These markers will include inflammatory cytokines, c-reactive protein, oxidative stress markers such as malondialdehyde, oxidised low-density lipoprotein, measures of antioxidant levels, markers of DNA damage (8-hydroxy-2-deoxyguanosine), and growth factors including insulin-like growth factor, epidermal growth factor and BDNF.

\section{DATA MANAGEMENT}

\section{Description of Analysis Sample}

The sample to be included in the analyses will be all the participants who give consent to participate in the study and who meet all inclusion criteria and no exclusion criteria. Participants must also complete at least one post-baseline visit for their data to be included in the analysis.

\section{Method of Statistical Analysis}

All analyses will be conducted in accordance with the ICH E9 statistical principles, and are based on all randomised participants with at least one post-baseline observation (intention to treat population). Primary analyses will be undertaken on an intention-to-treat basis, including all participants as randomised, regardless of treatment actually received. Mixed- model repeated measures (MMRM) analyses will be used to analyse change in MADRS score because it has been demonstrated to be superior to the last observation carried forward method for handling missing data in longitudinal designs. The MMRM model includes the fixed, categorical effects of treatment, investigator, visit, and treatment-by-visit interaction, as well as the continuous, fixed covariates of baseline score and baseline score-by-visit interaction. The
MMRM includes all available data at each time point. Results from the analysis of dichotomous data will be presented as proportions, with $95 \%$ confidence interval, and Fisher's exact $p$-value where appropriate. Non-parametric statistics will be used when assumptions for parametric methods are violated. Effect sizes will be calculated using Cohen's guidelines. All tests of treatment effects will be conducted using a two-sided alpha level of 0.05 and $95 \%$ confidence intervals. Significance will be defined as $p \leq$ 0.05 .

\section{Sample Size and Power}

A total of 60 participants meeting the DSM-IV-TR criteria for MDD, and experiencing moderate to severe illness symptomatology, will be recruited. The primary outcome measure will be change in MADRS score at week 12. For a two tailed analysis ${ }^{42)}$ with $\alpha=0.05, \mathrm{Z} \alpha=1.96$ and with $\beta=0.2, \mathrm{Z} \beta=0.842, \mathrm{~N}=60$ ( $\mathrm{n}=30$ in each arm), the study will be powered at $80 \%$ to detect a true difference in rating scale score between placebo and minocycline treated groups if the difference of the means is not less than 0.724 times the standard deviation in the control group (effect size).

An effect size of 0.4 or greater for difference in MADRS scores in randomised controlled trials of antidepressants versus placebo has been suggested as necessary to suggest clinical utility for an antidepressant used as monotherapy. Effect sizes of 0.28 to 0.51 have been reported for difference in MADRS score in trials of citalopram and escitalopram after 8 weeks of treatment. ${ }^{43)}$ This suggests that at $\mathrm{N}=60$ the proposed depression study may not be sufficiently powered to detect small effect size differences between placebo and minocycline treated groups for changes in MADRS score if the efficacy of minocycline is not greater than conventional antidepressant monotherapy. However, for a pilot study a sample size of 60 should be sufficient to demonstrate trends that would indicate a need for a larger trial.

\section{RESULTS AND DISCUSSION}

The study is currently being conducted at the two Victorian sites and has relevant approval from the Human Research and Ethics Committees. The study is planned to begin recruitment at the Thailand site in May 2014, with the relevant aprpovals in place.

The study has recruited 15 participants to date and is planning to complete the recruitment phase in 2014. 


\section{CONCLUSIONS}

Given the paucity of new therapies for unipolar depression and the burden the illness costs not only at a personal level, but for the wider community and the associated health-economics concerns, this trial is timely. The study may not only provide evidence of a novel therapy for unipolar depression, but may also assist in driving new targets of therapeutic pursuit through the exploration of biomarkers. Overall, we are hopeful that this study may have significant benefit for those with unipolar depression.

\section{Acknowledgments}

The authors would like to acknowledge the support of the Brain and Behavior Foundation, the Australasian Society of Bipolar and Depressive Disorders and the Alfred Deakin Postdoctoral Research Fellowship. We would also like to acknowledge the service support of Barwon Health, Deakin University, The Melbourne Clinic, The University of Melbourne and Chulalongkorn University.

\section{REFERENCES}

1. World Health Organization. The world health report 2001. Mental health: new understanding, new hope. Geneva: World Health Organization;2001.

2. Maes M, Yirmyia R, Noraberg J, Brene S, Hibbeln J, Perini $\mathrm{G}$, et al. The inflammatory \& neurodegenerative (I\&ND) hypothesis of depression: leads for future research and new drug developments in depression. Metab Brain Dis 2009; 24:27-53.

3. Schiepers OJ, Wichers MC, Maes M. Cytokines and major depression. Prog Neuropsychopharmacol Biol Psychiatry 2005;29:201-217.

4. Nishida A, Hisaoka K, Zensho H, Uchitomi Y, Morinobu $\mathrm{S}$, Yamawaki S. Antidepressant drugs and cytokines in mood disorders. Int Immunopharmacol 2002;2:1619-1626.

5. Peet M, Murphy B, Shay J, Horrobin D. Depletion of omega-3 fatty acid levels in red blood cell membranes of depressive patients. Biol Psychiatry 1998;43:315-319.

6. Maes M, De Vos N, Pioli R, Demedts P, Wauters A, Neels $\mathrm{H}$, et al. Lower serum vitamin E concentrations in major depression. Another marker of lowered antioxidant defenses in that illness. $J$ Affect Disord 2000;58:241-246.

7. Maes M, Christophe A, Delanghe J, Altamura C, Neels H, Meltzer HY. Lowered omega 3 polyunsaturated fatty acids in serum phospholipids and cholesteryl esters of depressed patients. Psychiatry Res 1999;85:275-291.

8. Bilici M, Efe H, Köroğlu MA, Uydu HA, Bekaroğlu M, Değer O. Antioxidative enzyme activities and lipid peroxidation in major depression: alterations by antidepressant treatments. J Affect Disord 2001;64:43-51.

9. Selley ML. Increased (E)-4-hydroxy-2-nonenal and asymmetric dimethylarginine concentrations and decreased nitric oxide concentrations in the plasma of patients with major depression. J Affect Disord 2004;80:249-256.

10. Khanzode SD, Dakhale GN, Khanzode SS, Saoji A, Palasodkar R. Oxidative damage and major depression: the potential antioxidant action of selective serotonin re-uptake inhibitors. Redox Rep 2003;8:365-370.

11. Forlenza MJ, Miller GE. Increased serum levels of 8hydroxy-2'-deoxyguanosine in clinical depression. Psychosom Med 2006;68:1-7.

12. Gałecki P, Gałecka E, Maes M, Chamielec M, Orzechowska A, Bobińska $\mathrm{K}$, et al. The expression of genes encoding for COX-2, MPO, iNOS, and SPLA2-IIA in patients with recurrent depressive disorder. J Affect Disord 2012;138: 360-366.

13. Maes M, Kubera M, Mihaylova I, Geffard M, Galecki P, Leunis JC, et al. Increased autoimmune responses against auto-epitopes modified by oxidative and nitrosative damage in depression: implications for the pathways to chronic depression and neuroprogression. J Affect Disord 2013;149: 23-29.

14. Allen SJ, Dawbarn D. Clinical relevance of the neurotrophins and their receptors. Clin Sci (Lond) 2006;110: 175-191.

15. Castrén E, Rantamäki T. The role of $B D N F$ and its receptors in depression and antidepressant drug action: Reactivation of developmental plasticity. Dev Neurobiol 2010;70:289297.

16. Otsuki K, Uchida S, Watanuki T, Wakabayashi Y, Fujimoto $\mathrm{M}$, Matsubara T, et al. Altered expression of neurotrophic factors in patients with major depression. J Psychiatr Res 2008; 42:1145-1153.

17. Berk M, Kapczinski F, Andreazza AC, Dean OM, Giorlando F, Maes M, et al. Pathways underlying neuroprogression in bipolar disorder: focus on inflammation, oxidative stress and neurotrophic factors. Neurosci Biobehav Rev 2011;35: 804-817.

18. Dean OM, Data-Franco J, Giorlando F, Berk M. Minocycline: therapeutic potential in psychiatry. CNS Drugs 2012; 26:391-401.

19. Kim SS, Kong PJ, Kim BS, Sheen DH, Nam SY, Chun W. Inhibitory action of minocycline on lipopolysaccharideinduced release of nitric oxide and prostaglandin E2 in BV2 microglial cells. Arch Pharm Res 2004;27:314-318.

20. Ahuja M, Bishnoi M, Chopra K. Protective effect of minocycline, a semi-synthetic second-generation tetracycline against 3-nitropropionic acid (3-NP)-induced neurotoxicity. Toxicology 2008;244:111-122.

21. Morimoto N, Shimazawa M, Yamashima T, Nagai H, Hara $\mathrm{H}$. Minocycline inhibits oxidative stress and decreases in vitro and in vivo ischemic neuronal damage. Brain Res 2005; 1044:8-15.

22. Homsi S, Federico F, Croci N, Palmier B, Plotkine M, Marchand-Leroux $\mathrm{C}$, et al. Minocycline effects on cerebral edema: relations with inflammatory and oxidative stress markers following traumatic brain injury in mice. Brain Res 2009;1291:122-132.

23. Orio L, Llopis N, Torres E, Izco M, O'Shea E, Colado MI. A study on the mechanisms by which minocycline protects against MDMA ('ecstasy')-induced neurotoxicity of 5-HT cortical neurons. Neurotox Res 2010;18:187-199.

24. Kernt M, Neubauer AS, Eibl KH, Wolf A, Ulbig MW, Kampik A, et al. Minocycline is cytoprotective in human trabecular meshwork cells and optic nerve head astrocytes by increasing expression of XIAP, survivin, and Bcl-2. Clin Ophthalmol 2010;4:591-604.

25. Hashimoto K, Ishima T. A novel target of action of minocycline in NGF-induced neurite outgrowth in PC12 cells: translation initiation [corrected] factor eIF4AI. PLoS One 2010;5:e15430. 
26. Homsi S, Piaggio T, Croci N, Noble F, Plotkine M, Marchand-Leroux $\mathrm{C}$, et al. Blockade of acute microglial activation by minocycline promotes neuroprotection and reduces locomotor hyperactivity after closed head injury in mice: a twelve-week follow-up study. J Neurotrauma 2010; 27:911-921.

27. Kim BJ, Kim MJ, Park JM, Lee SH, Kim YJ, Ryu S, et al. Reduced neurogenesis after suppressed inflammation by minocycline in transient cerebral ischemia in rat. J Neurol Sci 2009;279:70-75.

28. Liu Z, Fan Y, Won SJ, Neumann M, Hu D, Zhou L, et al. Chronic treatment with minocycline preserves adult new neurons and reduces functional impairment after focal cerebral ischemia. Stroke 2007;38:146-152.

29. Zhong J, Lee WH. Hydrogen peroxide attenuates insulinlike growth factor-1 neuroprotective effect, prevented by minocycline. Neurochem Int 2007;51:398-404.

30. Garcia-Martinez EM, Sanz-Blasco S, Karachitos A, Bandez MJ, Fernandez-Gomez FJ, Perez-Alvarez S, et al. Mitochondria and calcium flux as targets of neuroprotection caused by minocycline in cerebellar granule cells. Biochem Pharmacol 2010;79:239-250.

31. Kraus RL, Pasieczny R, Lariosa-Willingham K, Turner MS, Jiang A, Trauger JW. Antioxidant properties of minocycline: neuroprotection in an oxidative stress assay and direct radical-scavenging activity. J Neurochem 2005;94:819-827.

32. Zhang L, Kitaichi K, Fujimoto Y, Nakayama H, Shimizu E, Iyo M, et al. Protective effects of minocycline on behavioral changes and neurotoxicity in mice after administration of methamphetamine. Prog Neuropsychopharmacol Biol Psychiatry 2006;30:1381-1393.

33. Zhang L, Shirayama Y, Iyo M, Hashimoto K. Minocycline attenuates hyperlocomotion and prepulse inhibition deficits in mice after administration of the NMDA receptor antagonist dizocilpine. Neuropsychopharmacology 2007;32: 2004-2010

34. Molina-Hernández M, Téllez-Alcántara NP, Pérez-García J, Olivera-Lopez JI, Jaramillo-Jaimes MT. Desipramine or glutamate antagonists synergized the antidepressant-like actions of intra-nucleus accumbens infusions of minocycline in male Wistar rats. Prog Neuropsychopharmacol Biol Psychiatry 2008;32:1660-1666.

35. Deak T, Bellamy C, D'Agostino LG, Rosanoff M, McElderry NK, Bordner KA. Behavioral responses during the forced swim test are not affected by anti-inflammatory agents or acute illness induced by lipopolysaccharide. Behav Brain Res 2005;160:125-134.

36. Arakawa S, Shirayama Y, Fujita Y, Ishima T, Horio M, Muneoka K, et al. Minocycline produced antidepressant-like effects on the learned helplessness rats with alterations in levels of monoamine in the amygdala and no changes in BDNF levels in the hippocampus at baseline. Pharmacol Biochem Behav 2012;100:601-606.

37. Pae CU, Marks DM, Han C, Patkar AA. Does minocycline have antidepressant effect? Biomed Pharmacother 2008;62: 308-311.

38. Miyaoka T, Yasukawa R, Yasuda H, Hayashida M, Inagaki $\mathrm{T}$, Horiguchi J. Possible antipsychotic effects of minocycline in patients with schizophrenia. Prog Neuropsychopharmacol Biol Psychiatry 2007;31:304-307.

39. Miyaoka T, Yasukawa R, Yasuda H, Hayashida M, Inagaki $\mathrm{T}$, Horiguchi J. Minocycline as adjunctive therapy for schizophrenia: an open-label study. Clin Neuropharmacol 2008;31:287-292.

40. Levkovitz Y, Mendlovich S, Riwkes S, Braw Y, LevkovitchVerbin $\mathrm{H}$, Gal G, et al. A double-blind, randomized study of minocycline for the treatment of negative and cognitive symptoms in early-phase schizophrenia. J Clin Psychiatry 2010;71:138-149.

41. Levine J, Cholestoy A, Zimmerman J. Possible antidepressant effect of minocycline. Am J Psychiatry 1996;153: 582.

42. Stolley PD, Strom BL. Sample size calculations for clinical pharmacology studies. Clin Pharmacol Ther 1986;39:489490.

43. Bech P, Tanghøj P, Cialdella P, Andersen HF, Pedersen AG. Escitalopram dose-response revisited: an alternative psychometric approach to evaluate clinical effects of escitalopram compared to citalopram and placebo in patients with major depression. Int J Neuropsychopharmacol 2004;7:283-290. 